\title{
Axisymmetric and stationary magnetic field structures in neutron star crusts under various boundary conditions
}

\author{
Kotaro Fujisawa ${ }^{1}$ and Shota Kisaka ${ }^{2}$ \\ ${ }^{1}$ Department of Earth Science and Astronomy, Graduate School of Arts and Sciences, \\ University of Tokyo, Komaba, Meguro-ku, Tokyo 153-8902, Japan \\ email: fujisawa@ea.c.u-tokyo.ac.jp \\ ${ }^{2}$ Institute for Cosmic Ray Research, University of Tokyo, \\ 5-1-5 Kashiwa-no-ha, Kashiwa city, Chiba 277-8582, Japan
}

\begin{abstract}
We have calculated many Hall equilibrium states within the neutron star crust under various boundary conditions in order to investigate the influences of the boundary conditions clearly. We have found two important features of these solutions. First, the magnitude of the core magnetic fields affects the toroidal to total magnetic field energy ratio within the crust $\left(E_{t} / E\right)$. If the core magnetic fields are vanished, the crustal toroidal magnetic fields become weak and the typical energy ratio is only $E_{t} / E \sim 0.1 \%$. If the core magnetic fields are strong, however, the crustal toroidal magnetic fields become strong and the typical ratio reaches $E_{t} / E \sim 15 \%$. Second, the core toroidal magnetic fields and the twisted magnetosphere around the star make the size of the crustal toroidal magnetic field regions large. Therefore if the strong core magnetic fields have strong toroidal component, both strength and size of the crustal toroidal magnetic fields become large. These results show that the Hall MHD evolutions would be deeply affected by both inner and outer boundary conditions.
\end{abstract}

Keywords. Stars: interior - Stars: neutron - Stars: magnetic fields

\section{Introduction}

The decay of the magnetic field is considered as an important energy source of magnetar activity and neutron star heating, but the Ohmic diffusion cannot be effective physical mechanism for the decay because of the high electrical conductivity inside the neutron star. The Hall effect is considered as a key of the promotion of the magnetic fields decay (e.g. Goldreich \& Reisenegger 1992). Recently, Hall MHD numerical simulations (Kojima \& Kisaka 2012, Viganò et al. 2013) and Hall equilibrium states (Gourgouliatos et al. 2013) have been calculated. Since the boundary conditions are very important in both cases, we have calculated the Hall equilibria within the neutron star crust under various boundary conditions in order to investigate the influences of the boundary conditions.

\section{Results and Discussion}

We have obtained Hall equilibrium states (see Gourgouliatos et al. 2013) using Green function method with arbitrary current sheets (see Fujisawa \& Eriguchi 2013). For simplicity, we divided the magnetized neutron star interior into three regions in order to include the boundary conditions systematically. Each region is core $\left(0 \leqslant r \leqslant r_{s}\right)$, crust $\left(r_{i n} \leqslant r \leqslant r_{s}\right)$, vacuum or magnetosphere $\left(r \leqslant r_{s}\right)$ respectively. Here, $r_{i n}$ and $r_{s}$ denote the core-crust boundary radius and the stellar radius. Numerical results and the ratios $E_{t} / E$ are displayed in Fig. 1 and Fig. 2. 

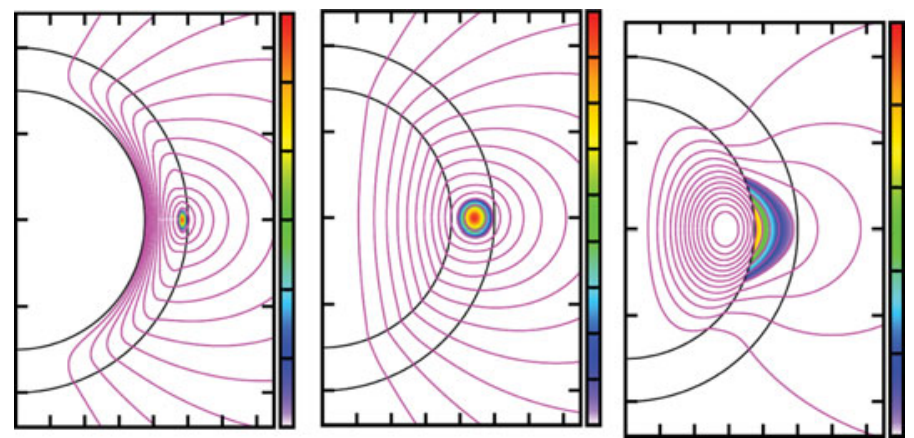

Figure 1. The poloidal magnetic fields configurations and the strength of the crustal toroidal magnetic fields (color maps). A pure crustal magnetic field model (left, $E_{t} / E \sim 0.5 \%$ ), a crustcore magnetic field model (center, $E_{t} / E \sim 15 \%$ ) and a very strong core magnetic field model (right, $E_{t} / E \sim 14 \%$ ) are displayed. The left model has the smallest $E_{t} / E$ and the center and the right models have larger $E_{t} / E$. The right model has the strong core toroidal magnetic field and the size of the toroidal magnetic field region is largest in these models.
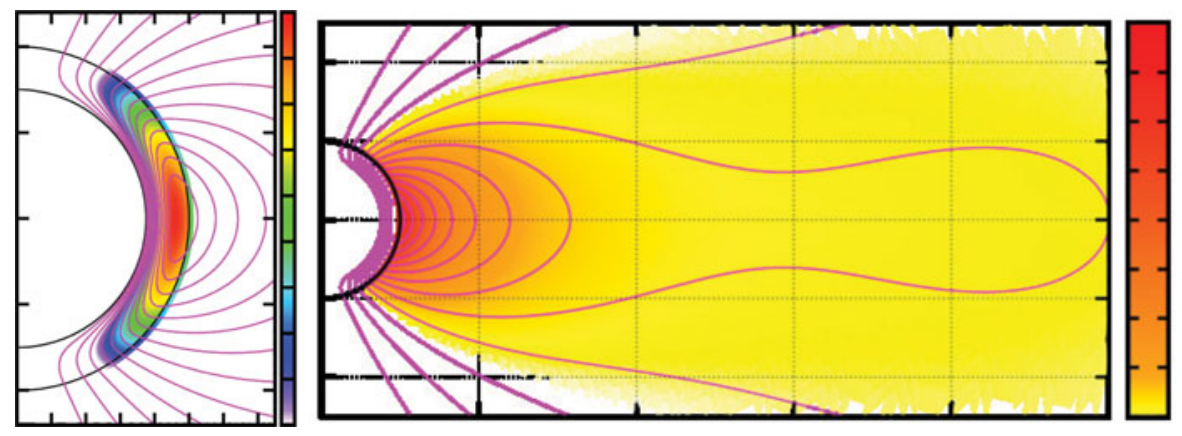

Figure 2. The crustal poloidal fields and the strength of the crustal toroidal fields (left) and the magnetospheric poloidal fields and the strength of the toroidal current density (right). The size of the crustal toroidal magnetic field region is much larger than those of models in Fig. 1.

As we have seen in these figures, the boundary conditions change the Hall equilibria within the crust significantly. These boundary conditions would affect the Hall drifts and evolutions deeply. Since the strong initial toroidal magnetic fields enforce the Hall drift (Kojima \& Kisaka 2012) during the Hall MHD evolution and the Hall drift would stop when the magnetic fields reach Hall equilibrium configurations, we can evaluate the efficiency of the Hall drift using the value of $E_{t} / E$ of our results. If we assume the strong initial toroidal fields, the strong core magnetic fields (right panel in Fig. 1) would weaken the Hall drift because $E_{t} / E$ is larger. In the pure crustal field case (left panel in Fig. 1), the Hall drift would become more active because $E_{t} / E$ is smallest in these models.

\section{References}

Fujisawa, K. \& Eriguchi, Y., 2013, MNRAS, 432, 1245

Goldreich, P. \& Reisenegger, A., 1992, ApJ, 395, 250

Gourgouliatos, K. N., Cumming, A., Reisenegger, A., Armaza, C., Lyutikov, M., \& Valdivia, J. A., 2013, MNRAS, 434, 2480

Kojima, Y. \& Kisaka, S., 2012, MNRAS, 421, 2722

Viganò, D., Rea, N., Pons, J. A., Perna, R., Aguilera, D. N., \& Miralles, J. A., 2013, MNRAS, 434,123 